\title{
Un estudio sobre la aprehensión conceptual de las inecuaciones
}

\author{
Mónica Campos \\ profesoramonicacampos1965@gmail.com \\ https://orcid.org/0000-0002-4260-6719 \\ Instituto Superior de Formación Docente Simón Bolívar \\ Córdoba, Argentina \\ Mabel Rodríguez \\ mrodri@campus.ungs.edu.ar \\ https://orcid.org/0000-0002-8425-8572 \\ Universidad Nacional de General Sarmiento \\ Los Polvorines, Argentina
}

Recibido: 11/03/2020 Aceptado: 25/04/2020

\section{Resumen}

En este artículo presentamos una investigación enmarcada en el Enfoque Cognitivo en Educación Matemática y la hemos desarrollado con estudiantes del primer año de la formación de profesores de matemática en una institución argentina. Inicialmente identificamos una problemática docente que podemos sintetizar como la falta de comprensión de algunos objetos matemáticos que se consideran básicos y base para la construcción de otros de mayor complejidad. A partir de esta inquietud, planteamos un problema de investigación que asume la teoría de las representaciones semióticas de Raymond Duval como marco teórico. El objetivo general fue adquirir conocimiento respecto de la aprehensión conceptual de estudiantes respecto del objeto matemático inecuaciones. Específicamente, nos propusimos describir los registros de representación semiótica que estudiantes de primer año ponen en juego al resolver inecuaciones y al expresar sus conjuntos solución y además describir el grado de aprehensión conceptual alcanzado luego de la enseñanza recibida. Diseñamos, fundamentamos y aplicamos un test para recabar datos y propusimos un análisis en dos dimensiones. Una de ellas nos permitió reconocer los registros utilizados en la resolución de cada uno de los ejercicios mientras que, la otra, nos permitió valorar la aprehensión conceptual alcanzada. Asimismo, el análisis por estudiante nos permitió advertir que varios de ellos se desempeñan, ante las resoluciones de inecuaciones, de maneras semejantes. Esos rasgos comunes fueron plasmados en la construcción de una rúbrica que deja de manifiesto tipos de desempeño que realizan estudiantes que han logrado la aprehensión conceptual en distintas etapas de desarrollo. Finalmente hemos retomado la problemática docente que originó la investigación y dejamos una serie de consideraciones para la enseñanza de las inecuaciones que resultarían clave para favorecer su comprensión.

Palabras clave: Aprehensión Conceptual de Inecuaciones. Teoría Semiótica. Inecuaciones. Registros de Representación Semiótica.

\section{Um estudo sobre a apreensão conceitual das inequações}

\section{Resumo}

Neste artigo, apresentamos uma pesquisa enquadrada na Abordagem Cognitiva em Educação Matemática e a desenvolvemos com alunos do primeiro ano de formação de professores de 
matemática em uma instituição argentina. Inicialmente, identificamos um problema de ensino que podemos sintetizar como a falta de entendimento de alguns objetos matemáticos considerados básicos e a base para a construção de outros de maior complexidade. Com base nessa preocupação, colocamos um problema de pesquisa que assume como referencial teórico a teoria das representações semióticas de Raymond Duval. O objetivo geral foi adquirir conhecimentos sobre a apreensão conceitual dos alunos sobre as desigualdades matemáticas dos objetos. Especificamente, propusemos descrever os registros de representação semiótica que os alunos do primeiro ano colocam em jogo ao resolver desigualdades e expressar seus conjuntos de soluções, além de descrever o grau de apreensão conceitual alcançada após o ensino recebido. Projetamos, suportamos e aplicamos um teste para coletar dados e propusemos uma análise bidimensional. Um deles nos permitiu reconhecer os registros utilizados na resolução de cada um dos exercícios, enquanto o outro nos permitiu avaliar a apreensão conceitual alcançada. Da mesma forma, a análise por aluno permitiu perceber que vários deles realizam, de maneira semelhante, a resolução de desigualdades. Essas características comuns foram refletidas na construção de uma rubrica que revela tipos de desempenho realizados por estudantes que alcançaram apreensão conceitual em diferentes estágios de desenvolvimento.

Finalmente, voltamos aos problemas de ensino que deram origem à pesquisa e deixamos uma série de considerações para o ensino de desigualdades que seriam fundamentais para favorecer sua compreensão.

Palavras Chave: Apreensão Conceitual das inequações. Teoria semiótica. Inequações. Registros de Representação Semiótica.

\title{
A study on the conceptual apprehension of inequalities
}

\begin{abstract}
In this article we present a research framed in the Cognitive approach in Mathematical Education and we worked with students of the first year of the training of mathematics teachers in an argentine institution. Initially, we identified a teaching problem that we can synthesize as the lack of understanding of some mathematical objects that are considered basic and the basis for the construction of others of greater complexity. Based on this concern, we pose a research problem that assumes Raymond Duval's theory of semiotic representations as a theoretical framework. The general objective was to acquire knowledge regarding the conceptual apprehension of students regarding the mathematical object inequalities. Specifically, we pretend to describe the registers of semiotic representation that first-year students use when solving inequalities and expressing their solution sets, and also describing the degree of conceptual apprehension achieved after the teaching received. We designed, supported and applied a test to collect data and proposed a two-dimensional analysis. One of these dimensions allowed us to recognize the semiotic registers used in solving each of the exercises, while the other allowed us to assess the conceptual apprehension achieved by the students. Likewise, the analysis per student allowed us to notice that several of them perform, in the face of the resolution of inequalities, in similar ways. These common features were reflected in the construction of a rubric that reveals types of performance carried out by students who have achieved conceptual apprehension at different stages of development.

Finally, we have returned to the teaching problem that originated the research and we leave a series of considerations for teaching inequalities that would be important to be considered for its understanding.
\end{abstract}


Keywords: Conceptual Apprehension of Inequalities. Semiotic Theory. Inequalities. Registers of Semiotic Representation.

\section{Introducción}

En Argentina, los índices de reprobación en matemática son muy altos y las críticas son continuas por los escasos conocimientos alcanzados por los estudiantes tanto en el nivel secundario como en el primario.

En el paso de la enseñanza secundaria a la superior, estas críticas se manifiestan en forma cruda y el nivel de conocimiento inicial es considerado uno de los factores que inciden de forma negativa sobre el proceso de aprendizaje de la matemática. Esta falta de conocimientos previos ocasiona, en la formación superior, la desaprobación de materias iniciales y este hecho, a su vez, suele ser una de las tantas causas de abandono o repitencia. El caso de las materias del primer año del Instituto Superior de Formación Docente Simón Bolívar de Córdoba Capital, Argentina, en donde se dicta la carrera de Profesorado de Educación Secundaria en Matemática, no son la excepción.

El equipo de profesores presenta una problemática enmarcada en un problema docente que se sintetiza como la falta de comprensión de algunos objetos matemáticos que se consideran básicos y base para la construcción de otros de mayor complejidad. Entre ellos se mencionan usualmente: la falta de dominio de operatoria básica, tanto numérica como algebraica, la falta de comprensión de las ecuaciones y desconocimiento de técnicas para su resolución, el desconocimiento de las funciones elementales, falta de comprensión del lenguaje simbólico, entre otros. Muchos de los objetos sobre los cuales los docentes advierten falta de conocimientos previos han sido estudiados en el nivel medio. Sin embargo, el estudio de inecuaciones suele no estar presente en la secundaria, su primer acercamiento se da en el nivel superior, y también la situación resulta preocupante. Su enseñanza se organiza de manera cuidadosa dado que se reconoce su potencialidad como base para el aprendizaje de otros saberes; sin embargo, los resultados no son significativamente diferentes a otros contenidos trabajados en el nivel anterior. Es decir, el equipo docente considera que los estudiantes tampoco logran comprender esta noción aunque hayan tenido a cargo su enseñanza.

Nuestras primeras inquietudes para el planteo de la investigación que reportamos en este artículo tomaron en consideración el problema docente recién mencionado. Para nuestra investigación consideramos la abstracción de los objetos matemáticos y su consecuente 
necesidad de emplear diversas representaciones para asimilarlos y aprehenderlos en su complejidad. Los símbolos, gráficos, números, palabras son usados como medios semióticos y todos ellos juegan un papel central en el desarrollo del pensamiento matemático. De este modo, y posicionándonos desde una perspectiva cognitiva, asumimos como punto de partida que para desarrollar la comprensión de las nociones matemáticas se requiere manipular representaciones semióticas.

En particular, los estudiantes que cursan en primer año espacios curriculares como Problemáticas de Álgebra o del Análisis Matemático del Profesorado de Educación Secundaria en Matemática en el Instituto Simón Bolívar, Córdoba Capital, tienen dificultades para reconocer un mismo objeto matemático en diferentes registros de representación. Indagar cómo se pone en juego, en estos estudiantes, el uso de distintas representaciones, podría ser una clave para poder explicar, desde la perspectiva cognitiva asumida, la falta de comprensión advertida. Hemos seleccionado las inecuaciones dado que su dominio les favorecerá, a los estudiantes, la comprensión de otros conceptos matemáticos propios del nivel superior, y porque podríamos capitalizar los resultados del estudio para favorecer su enseñanza en el Profesorado.

El objetivo general del trabajo que aquí presentamos es adquirir conocimiento sobre la aprehensión conceptual del objeto inecuación. Damos precisiones teóricas y metodológicas del planteo del problema de investigación en el siguiente apartado.

\section{Antecedentes y planteo del problema de investigación}

En campos del conocimiento como la biología, anatomía, etc. los objetos que se estudian pueden ser percibidos de alguna manera por los sentidos, pero en matemática el acceso es necesariamente semiótico, por medio de algún tipo de representación. Esta distinción, que es inherente a la matemática, fue el puntapié inicial para importantes trabajos de investigación de muchos autores interesados en mejorar los aprendizajes de sus estudiantes. Presentamos un breve recorrido, parte de un Estado del Arte más amplio (Campos, 2020) que iniciamos a partir de los aportes de Raymond Duval en 1993, considerado hoy en día como impulsor de estudios de índole semiótico.

En 1993, Duval publicó un artículo advirtiendo la importancia de distinguir el objeto matemático de sus representaciones, pero a la vez alertando de la complejidad de que un sujeto comprenda un objeto, abstracto, al que nunca logrará acceder excepto a través de sus representaciones. Otros llamaron a este último hecho la Paradoja de Duval (por ejemplo, 
D’Amore, Fandiño, Iori y Matteuzzi, 2015). Duval (1995) propone las primeras caracterizaciones semiótico-cognitivas de los objetos matemáticos. Caracteriza al objeto matemático como un invariante operatorio o lógico-discursivo de una variada cantidad de posibles representaciones y establece que un indicador posible del acceso a ese conocimiento podría ser su reconocimiento en al menos dos tipos de representaciones.

Cada sistema semiótico presenta limitaciones y reglas propias de funcionamiento y las representaciones semióticas juegan un rol primordial en la constitución cognitiva del pensamiento. Un sistema semiótico es considerado un registro de representación cuando tiene la facultad de describir, de permitir identificar al objeto que quiere representar, contiene los rasgos y datos necesarios que permiten dicha representación. A la vez, debe brindar la posibilidad de tratamiento. Esto significa la transformación de una representación a otra en el mismo registro en el que está siendo trabajada. Otra facultad importante de un sistema semiótico es que debe permitir algún tipo de conversión. Siguiendo con Duval (1995), una conversión es la transformación de una representación propia de un registro a otra representación del objeto matemático en otro registro de representación. Es decir, es una transformación externa. El lenguaje natural, las representaciones simbólicas, numéricas y gráficas son entendidos como diferentes tipos de registros.

Este aporte de Duval a la Educación Matemática sin dudas ha sido, y sigue siendo, muy valioso. Es utilizado y referenciado por distintos investigadores quienes coinciden que distinguir y coordinar distintos registros es una actividad necesaria y natural en matemática (Guzmán, 1998).

Siguiendo con los antecedentes, y centrando la atención en trabajos relacionados a las funciones, Guzmán (1988) se propuso poner en evidencia el rol que juegan los registros de representación en respuestas de estudiantes de primer año de la carrera de ingeniería en la Universidad de los Lagos, Chile. En el trabajo se plantean preguntas abiertas en lenguaje natural, otras en lenguaje simbólico y se incluyen en ellas representaciones gráficas. Si bien la conclusión de alguna manera verifica posibles supuestos, la autora expresa que en general los estudiantes reconocen los conceptos en lo que ella llama un monoregistro, es decir mediante una única forma de representación. Los estudiantes no pueden coordinar dos tipos de registros diferentes y para dar las respuestas privilegian el registro algebraico. 
Moreno \& Laborde (2003) muestran los resultados de un trabajo en el que estudian el cambio de registro con el uso de un software que permite un mayor grado de visualización. Plantearon a estudiantes proponer una modelización en la que debieron cambiar del registro verbal al algebraico. Los autores afirman que como los estudiantes recibían una enseñanza centrada en el registro algebraico, les resultó dificultoso, aún con el uso del software, la conversión a otro registro. Esta conclusión refuerza la hipótesis formulada por Duval (1988): la razón profunda de estas dificultades no se encuentra en los conceptos matemáticos, pero sí en el desconocimiento de las reglas de correspondencia semiótica entre el registro de representación gráfica y el simbólico.

En la Universidad Nacional de Mar del Plata, Argentina, en los primeros años de las carreras de ingeniería, se realizó un trabajo de investigación que tuvo por objetivo determinar cuáles son los registros de representación más adecuados para el estudio de subconjuntos de números complejos. Las investigadoras Aznar, Distéfano, Figueroa \& Moler (2010) plantean que extraer información de una representación gráfica no es un recurso habitual utilizado por los estudiantes. Esto refuerza la conclusión del estudio anterior de que el registro que se privilegia es el algebraico.

Jesús Macías Sánchez, en su tesis doctoral (2016) en la Universidad Complutense de Madrid, España, realiza una investigación que pone de manifiesto el lugar que ocupa la conversión de sistemas de representación semiótica en el currículo de nivel medio. Esta investigación nos aporta información relevante respecto de qué se prescribe para la enseñanza en el nivel educativo anterior al superior. Si bien la información es de España, podemos tomarla como referencia. El autor llegó a la conclusión que la noción de cambio de registros semióticos goza de invisibilidad didáctica (Macías, 2016), que no se atiende explícitamente desde la enseñanza y que solo se contemplan los tratamientos con mayor relevancia y prioridad. Asimismo expresa:

En menor proporción, el análisis muestra como ciertas conversiones aparecen en las leyes de manera explícita, por lo que podría parecer que existe cierta introducción al uso y manejo de más de un registro de representación para un determinado concepto. No obstante, podemos decir, a partir de la manera en que se enuncian y recogen, que es limitado y paupérrimo con respecto a las posibilidades existentes en ambas etapas educativas, pues más que favorecer un aprendizaje significativo e integral de los objetos matemáticos y las propiedades que los 
caracterizan, persiguen que el alumno disponga de herramientas de resolución de tareas, pasando por alto la complejidad existente en el proceso de relación entre representaciones por la falta de congruencia entre las unidades significantes que caracterizan a cada registro semiótico (Macías, 2016, p. 1032).

Pasar por alto la complejidad de los procesos de cambio de registro en los cursos superiores de la escuela media, puede ser una de las causas de las dificultades cuando los estudiantes abordan los objetos matemáticos en el nivel superior. Otra conclusión muy interesante de esta investigación es el estudio que realizan de la conversión de registros en los libros de texto a los que acceden los estudiantes de nivel medio. En este tipo de actividades la conversión de registros la hace el autor del libro y la actividad que el estudiante debe realizar se centra en los tratamientos solicitados en el registro que ya fue determinado por él. Al respecto Macías escribe:

El análisis mostró como los libros de texto promueven un uso simultáneo, no controlado, y en diversas ocasiones carente de sentido y funcionalidad, de algunos registros de representación semiótica, dando por hecho que el estudiante es capaz de interpretar y establecer relaciones entre ellas por sí mismo. Este hecho, junto con los obstáculos derivados por la falta de congruencia entre los diferentes registros semióticos que pueden entrar en juego en la conversión, genera la aparición de dificultades en el alumno debido tanto a la complejidad que ello conlleva como a la falta de destreza a la hora de articular varios sistemas de representación para un mismo concepto (Macías, 2016, p. 1034).

En estos libros de texto de nivel medio el autor encuentra muy usados los registros verbal, numérico y algebraico. Asimismo menciona que los registros gráficos y geométricos son usados como soporte de los anteriores y no como representaciones posibles de objetos matemáticos para los cuales resultaría la mejor opción.

Mabel Panizza, en su tesis doctoral se ocupa de "Las transformaciones semióticas en los procesos de definición de objetos matemáticos" (Panizza, 2018). En este trabajo la autora estudia cómo se manifiestan en los procedimientos de los estudiantes las operaciones de observación, reconocimiento, descripción y denominación, constitutivas de los procesos de definición de objetos matemáticos. Una de las conclusiones de este estudio es que los estudiantes no conciben espontáneamente ejemplos representativos de un objeto matemático, razón por la cual las operaciones antes mencionadas se realizan en muy pocas representaciones que tienen la 
marca de lo particular. Investigó las relaciones entre las operaciones de observación y descripción, de objetos matemáticos en escritura simbólica y o lenguaje natural, estableciendo que:

Las descripciones de propiedades de los objetos matemáticos en escritura simbólica y/o en lenguaje natural han mostrado en este Estudio Empírico otro fenómeno ligado a la relación entre las operaciones de observación y descripción. A menudo, las descripciones realizadas -en lenguaje natural o simbólico- son adecuadas a los objetos observados, pero no los caracterizan, en el sentido de que otros objetos caen dentro del alcance de la descripción (Panizza, 2018, p.207).

También plantea la existencia de una falta de articulación de diferentes registros semióticos dado que verificó que los estudiantes interpretan estas escrituras como si fueran caracterizaciones de un conjunto de objetos observados, o como nuevas descripciones que se pudieran obtener a partir de la conversión entre los diferentes tipos de registros. Panizza también menciona el problema didáctico de crear o encontrar las condiciones para que las conversiones surjan como estrategias propias de los estudiantes, según las necesidades creadas por las tareas a resolver, que le pudieran proponer los profesores. Esta es la capacidad que caracteriza los procesos del experto en la resolución de problemas (Panizza, 2018, p.130). La autora nos explica:

Las conversiones implícitamente solicitadas por una tarea o las que no son solicitadas ni implícita ni explícitamente requieren poner en juego estrategias de funcionamiento cognitivo superiores, en la medida en que -como analizamos anteriormente- en el primer caso suponen la elección de un registro pertinente para hacer una conversión y en el segundo suponen que se haya descubierto que la conversión entre registros es un instrumento para resolver cierto tipo de tareas. (Panizza, 2018, p.139)

Duval realiza lo que él llama "Un análisis cognitivo de los problemas de comprensión en el aprendizaje de las matemáticas" (Duval, 2016, p.61). En él, ratifica que la importancia de las representaciones en el aprendizaje de la matemática radica en el hecho de que se constituyen el único medio para el acceso a los objetos matemáticos. Lo que el autor llama estrategias matemáticas, implican de manera insoslayable la transformación de representaciones semióticas. Para tales transformaciones se debe considerar como absolutamente necesaria la coordinación de diferentes representaciones del objeto matemático.

Particularmente, como hemos mencionado anteriormente, la conversión de las representaciones semióticas constituye la actividad cognitiva menos espontánea y más difícil de 
adquirir para la mayoría de estudiantes y esta falta de coordinación entre los diferentes registros genera un obstáculo para los aprendizajes conceptuales.

Respecto específicamente de investigaciones que tomen el concepto de inecuación, encontramos el trabajo de Vancken, Engler y Müller (2010) quienes describen problemas que tienen los estudiantes a la hora de reconocer ecuaciones e inecuaciones. Concluyen que el gran problema que se plantea en el trabajo con inecuaciones es la ausencia de significado que este concepto tiene para los estudiantes. La raíz de esa falta de significado sería que su enseñanza suele reducirse al desarrollo de determinados métodos de resolución, no incluir la explicación de a qué métodos se refieren ni en qué se basan los mismos para realizar esas aseveraciones.

Nora Gatica, de la Universidad Nacional de San Luis (Argentina) y Alexander Maz Machado, de la Universidad de Córdoba (España), publican un trabajo el año 2012 en el que plantean que las inecuaciones, por más que están presentes en el curriculum de la escuela media en la Argentina, no es considerado un tema de importancia por los profesores de nivel medio ni de la Universidad. A la vez dicen también que los alumnos logran aprender a resolver inecuaciones a través de procedimientos y de reglas previamente establecidas, pero no enlazan aspectos conceptuales, por lo que el desarrollo de este conocimiento es "principalmente de carácter procedimental” (Gatica \& Maz Machado, 2012, p.2). En este trabajo se investigó cómo el concepto de inecuación fue construido por los estudiantes y cómo realizaron cambios de registros. Además de resultados específicos, observaron que los estudiantes no consiguen encontrar acabadamente el conjunto solución de la inecuación planteada sin intervención del profesor. Expresan además, que es esencial que se propongan tareas de conversiones entre los diferentes registros de representación y que deban interpretar resultados en uno y otro registro, tareas que no son frecuentes en los manuales escolares ni en las clases de matemática.

Garrote, Hidalgo y Blanco (2004) realizan un trabajo con estudiantes de primer año de bachillerato. Sus conclusiones pueden ser extrapolables a estudiantes de primer año del nivel superior. Los autores dicen que la comprensión del concepto de inecuación es deficiente en una parte importante de alumnos y que no establecen diferencias significativas entre este concepto y el de ecuación. Por otra parte mencionan que solamente reconocen que para las ecuaciones se utiliza el signo "=" mientras que en las inecuaciones se utilizan los signos " $<$, " $>$ ", “ $\leq$ " 0 " $\leq$, y solo lo reconocen como un nexo entre los dos miembros de ella. Una consecuencia de esta ausencia de sentido la constatan en las dificultades para la lectura en uno u otro caso. También 
presentan problemas cuando deben realizar conversiones del registro verbal al simbólico que incluye una doble desigualdad. En relación a los diferentes sistemas de representación encontraron que sus estudiantes solo usaban el registro simbólico hipotetizando que esto es consecuencia de las prácticas de enseñanza en el aula. Resaltan como problema de relevancia la ausencia de significado en el trabajo con inecuaciones, quedando la enseñanza de este contenido reducido a las técnicas algebraicas de resolución.

Alvarenga (2005), por su parte, presenta un conjunto de "construcciones mentales o esquemas, que el estudiante puede desarrollar con el fin de comprender el concepto de inecuación” (p.10). El autor explica en sus conclusiones que:

La interpretación es fundamental para el éxito en la resolución de situaciones que involucren inecuaciones, y debe incluir:

- La comprensión del conjunto solución y del significado de la variable;

- La identificación de lo que no puede ser solución;

- La identificación de las expresiones algebraicas que aparecen en la inecuación con funciones y análisis de la variación de las imágenes de esas funciones;

- La interpretación gráfica, o sea, la visualización de la inecuación por medio de gráficos de funciones;

- La comprensión del signo de desigualdad; y

- La identificación de las propiedades empleadas y de las respectivas transformaciones ocurridas en la inecuación y en su conjunto solución.

Otra idea interesante es la que plantea referida a lo que llama resolución gráfica. Menciona que la posibilidad o no de solución depende de las conexiones con otros contenidos y de "competencias matemáticas importantes que no han sido trabajadas en la enseñanza y el aprendizaje de las inecuaciones" (Alvarenga, 2005, p.140). Agrega que hay propuestas que analizan las inecuaciones por medio de gráficos, pero solamente cuando presentan las expresiones algebraicas, que son aisladas de otros tipos de resoluciones y que no hacen conexiones con otros contenidos, solo con funciones. Esto lo podemos interpretar como que no se presenta el registro gráfico como medio de representación ni de solución de las inecuaciones. Explica también que los alumnos presentan mucha dificultad en la interpretación y resolución gráfica de una inecuación (Alvarenga, 2005) y lo atribuye a la mala comprensión del concepto de función. 
Como podemos advertir, (enmarcado en el período 2004 a 2012) hay coincidencia en la falta de sentido construido por los estudiantes y la prevalencia de solo de métodos procedimentales de resolución de inecuaciones.

Este Estado del Arte nos permite seleccionar el enfoque que constituye el Marco Teórico de esta investigación. Consideramos la perspectiva de Duval (1998) dado que como se ha mostrado con los trabajos de Panizza (2018) y Aznar et al. (2010), queda de manifiesto la vigencia de las preocupaciones alrededor de los conceptos relacionados a las transformaciones semióticas y sus implicaciones en los aprendizajes matemáticos de los estudiantes. Señalamos en primer lugar que Duval, en sus distintos textos, utiliza los términos aprendizaje, comprensión y aprehensión conceptual. No encontramos precisiones en cuanto a los significados que el autor le atribuye a cada uno. Por ese motivo, consideramos que los utilizó con el significado del diccionario y no como elementos teóricos de su teoría.

El contexto de trabajo fue la asignatura Elementos de la Aritmética y el Álgebra que se encuentra en el primer año del Profesorado. En particular, nos ubicamos en la unidad sobre Expresiones Algebraicas que incluye los temas Ecuaciones y Sistemas de Ecuaciones, Inecuaciones y Sistemas de Inecuaciones. En la asignatura mencionada, las inecuaciones se les plantean a los estudiantes en registro algebraico. No pretendimos incidir sobre la enseñanza en este estudio, por lo que contemplamos este hecho como un punto de partida para el planteo de los objetivos de la investigación que son: (1) Describir las resoluciones de estudiantes de inecuaciones en términos de los registros de representación semiótica utilizados, conversiones y/o tratamiento; (2) Identificar registros de representación utilizados en la presentación de los conjuntos solución de inecuaciones y (3) Describir la aprehensión conceptual alcanzada sobre el objeto inecuación, sobre su resolución y sobre el conjunto solución.

Propusimos como instrumento para recabar datos un test que constara de inecuaciones para resolver. En la siguiente sección presentamos los criterios para diseñarlo, en qué términos fundamentamos su diseño e indicamos detalles del trabajo de campo y sistematización y análisis de datos.

\section{Desarrollo}

Respecto del diseño del test, formulamos cada una de las actividades que lo componen de manera que cumplieran los siguientes criterios: hubiera inecuaciones presentadas en todos los registros de representación (algebraico, numérico, verbal y gráfico); que sea posible expresar 
el conjunto solución en distintos registros y que admitan distintas resoluciones que puedan evidenciar tratamientos o conversiones. Atendimos a que el test cubra, de manera exhaustiva la presencia de los distintos registros tanto en la formulación de las consignas como en la expresión del conjunto solución. Decidimos no presentar consignas en el registro numérico porque entendemos que resultaría forzado y se perdería el sentido. El test finalmente quedó conformado por siete actividades. Incluimos a continuación la fundamentación de dos de ellas, con la intención de mostrar la profundidad en el detalle de análisis que nos determinó su selección para poder alcanzar los objetivos. (El análisis completo está en Campos, 2020).

Ejemplo 1: Fundamentación de la consigna del test 1c)

1c) Resolver $7+x^{2} \leq 7$

Las posibles resoluciones que anticipamos son las siguientes.

Primera resolución: en registro verbal. Resulta de analizar que el único número que sumado a 7 resulta menor o igual a 7 es el 0 .

Segunda resolución: en registro numérico. Probar con algunos valores numéricos hasta advertir que cualquier número positivo o negativo elevado al cuadrado sería positivo con lo que al sumar 7 siempre resultará mayor a 7 y no se cumpliría la desigualdad. La única posibilidad que queda analizar es la del 0, que cumple lo pedido.

Tercera resolución: en registro algebraico

$7+x^{2} \leq 7 \Leftrightarrow x^{2} \leq 7-7 \Leftrightarrow x^{2} \leq 0 \Leftrightarrow \sqrt{x^{2}} \leq \sqrt{0} \Leftrightarrow|x| \leq 0$. A partir de aquí, o se convierte al registro verbal y se concluye que el único valor que verifica es 0 , o se prosigue con el tratamiento algebraico, llegando al mismo resultado. 
Cuarta resolución: en registro gráfico

Interpretar la inecuación en registro gráfico implica encontrar los valores reales $x$ para los cuales la función $h: \mathbb{R} \rightarrow \mathbb{R}, h(x)=7+$ $x^{2}$ tiene su gráfico igual o por debajo de $g: \mathbb{R} \rightarrow \mathbb{R}, g(x)=7$. Entonces se interpreta el planteo como "identificar los valores de la variable independiente para los cuales la parábola que describe la función cuadrática es menor o igual la recta $y=7$ ', hay solo un

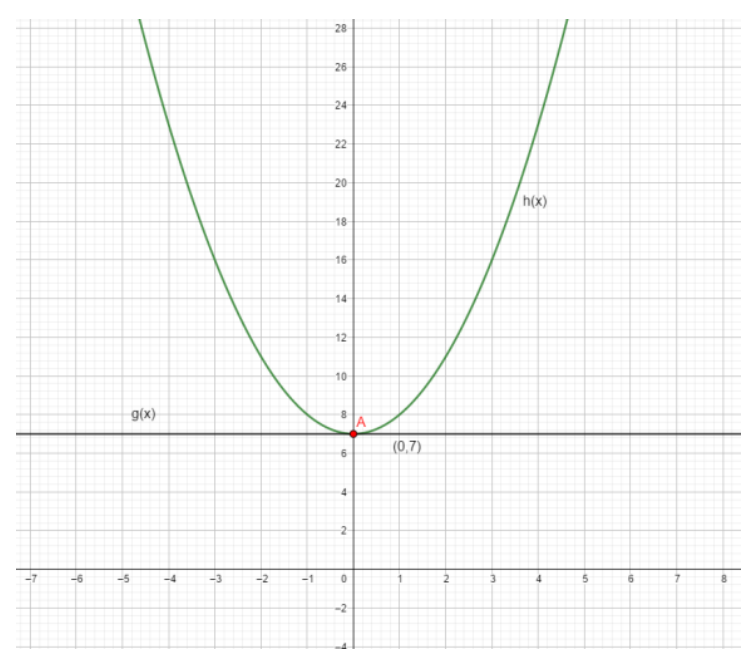
punto en el que son iguales: el cero.

El conjunto solución, en cualquiera de los casos, puede ser presentado en los siguientes registros. Numérico $S=\{0\}$ o solo 0 (no sería una notación correcta, pero posible).

Simbólicamente: $\{x \in \mathbb{R},|x|=0\}$

Gráfico en la recta real:

Ejemplo 2: Fundamentación de la consigna 2 del test.

La misma tiene dos ítems que presentamos separadamente.

2a) Resolver sen $x \leq 1$

La consigna está planteada en el registro algebraico y fue seleccionada dado que no admite tratamiento en este registro. Es necesario plantear una conversión a algún otro registro.

Primera resolución: conversión al registro gráfico.

Hay que identificar los valores de la variable independiente $x$ para los cuales la función $f: \mathbb{R} \rightarrow \mathbb{R}, \quad f(x)=\operatorname{sen} x \quad$ se mantiene menor o igual a 1 . Para ello se podría graficar $f(x)=\operatorname{sen} x \quad \mathrm{y}$ $g: \mathbb{R} \rightarrow \mathbb{R}, g(x)=1$. A partir de allí el análisis debe ser desde el gráfico y sobre el eje de las abscisas.

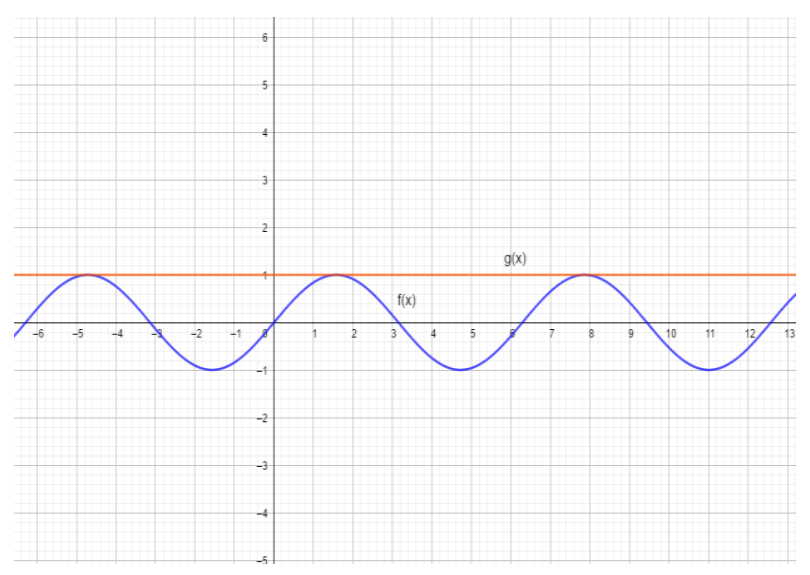


Segunda solución: en registro gráfico y numérico

Es posible graficar la circunferencia trigonométrica y comparar en ella la medida del segmento que representa al seno del ángulo respecto de 1 (medida del radio de la circunferencia).

Puede resultar una resolución en registro numérico pero necesita de la conversión desde el planteo algebraico, al gráfico y al numérico.

El gráfico es solo ilustrativo, los estudiantes no contarán con computadoras a la hora de realizar el test.
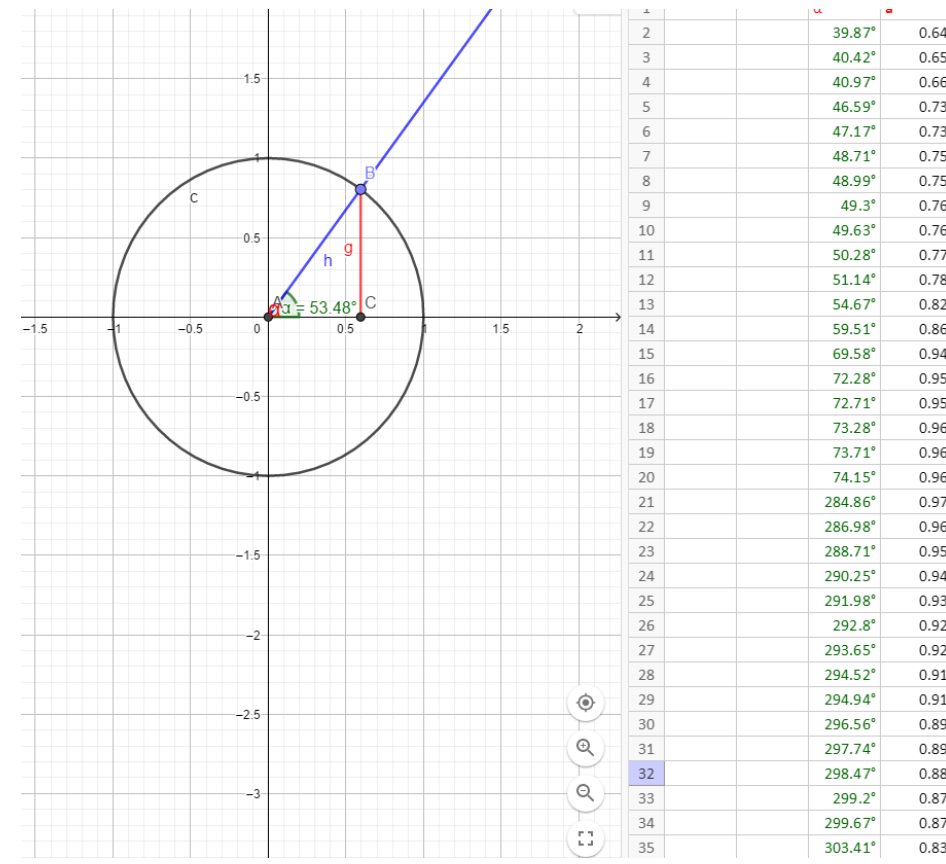

Tercera solución: en registro numérico.

Dar valores a la variable independiente y hacer cuentas con la calculadora. Sin tener forma de argumentar de un modo correcto, matemáticamente hablando, suponer que el conjunto solución son todos los números reales.

Las posibles presentaciones del conjunto solución, ante cualquier resolución, son:

En el registro verbal, para todos los números reales se cumple que sen $x$ es siempre menor o igual a 1 .

Numérico: $\{\ldots-10 \ldots \ldots-1 \ldots 0 \ldots 1 \ldots 2 \ldots 8 \ldots\}$ (no correcto, pero posible)

Algebraico: $\mathbb{R}$

Gráfico:

2b) Resolver sen $x \geq 1$

Las resoluciones son análogas, solo cambia el conjunto solución que en este caso es infinito numerable y requiere ser expresado adecuadamente. Solo incluimos posibles formas de representarlo. 
En el registro verbal el conjunto solución está formado por todos aquellos valores de $x$ que se obtienen sumándole a $\frac{\pi}{2}$ múltiplos enteros de $2 \pi$. También se puede pensar como la suma de $\frac{\pi}{2}$ más el doble de un múltiplo entero de $\pi$.

El conjunto solución en el registro numérico: $\left\{\ldots .-\frac{7}{2} \pi,-\frac{3}{2} \pi, \frac{1}{2} \pi, \frac{5}{2} \pi, \frac{9}{2} \pi, \frac{11}{2} \pi \ldots\right\}$ o bien $\{\ldots-11,-1.47,1.57,7.85,14.14,20.42 \ldots$.$\} con valores aproximados.$

La generalización puede expresarse

$$
\{\mathrm{x} \in \mathbb{R}, \mathrm{x}=(4 \mathrm{k}-3) \pi / 2, \text { con } \mathrm{k} \in \mathbb{Z}\} \circ\left\{x \in \mathbb{R}, x=\frac{\pi}{2}+k .2 \pi \text {, con } k \in \mathbb{Z}\right\}
$$

en registro algebraico.

El trabajo de campo fue realizado con 41 estudiantes, sobre un total de 50, de la asignatura Elementos de la Aritmética y el Álgebra, de manera presencial en dos horas dentro del horario de sus clases.

Para realizar el análisis de los datos recabados, primeramente codificamos los estudiantes, para preservar su identidad y generamos una serie de referencias y tablas en las que volcamos los datos. Especificamos los registros de representación que fueron utilizados en las resoluciones, denotando: V (verbal), N (Numérico), NT (Numérico con una tabla de valores), A (Algebraico), GR (Gráfico en $\mathbb{R})$ y GP $\left(\right.$ Gráfico en $\left.\mathbb{R}^{2}\right)$. Por otra parte, para expresar el conjunto solución utilizamos las denominaciones: NE (Numérico: presentar exhaustivamente los valores), NI (Numérico, con notación de intervalos), V (Verbal), AC (Algebraico, notación de conjunto), AI (Algebraico con notación de intervalo), AD (Algebraico, en términos de desigualdad), GR (Gráfico en $\mathbb{R})$ y GP (Gráfico en $\mathbb{R}^{2}$, sobre el eje x). A su vez registramos si responde bien, regular, mal, o no responde.

Con esos datos en el cuadro, realizamos dos tipos de análisis: uno por ejercicio y otro por estudiante. Mientras que la mirada por ejercicio nos permitió describir las resoluciones que los estudiantes realizaron de las inecuaciones y la expresión del conjunto solución en términos de registros de representación, el análisis por estudiante nos permitió dar cuenta de la aprehensión conceptual alcanzada sobre el objeto inecuación. Con este último pudimos encontrar características comunes y construir una rúbrica que puede ser utilizada para valorar el desarrollo en la comprensión del objeto matemático "inecuaciones". 


\section{Resultados y discusión}

\section{Respecto del análisis por ejercicio}

En todos los casos, cuando encontramos errores matemáticos (sea de planteo, operatoria numérica o algebraica), nos interesó analizar la coherencia entre su resolución y la respuesta dada, con la finalidad de poder decidir si estaban interpretando la inecuación.

El ejercicio 1a) plantea resolver $2(x-1)(x+2)<0$. Las resoluciones recibidas nos permiten plantear dos casos: quienes "interpretaron que el planteo es, efectivamente, una inecuación" y quienes "consideraron el planteo como si fuera el de la ecuación asociada". Respecto de los registros de representación semiótica utilizados en las resoluciones de los estudiantes, se presentaron dos tipos de tratamientos generales, uno que considera únicamente la expresión cuadrática y el que no la usa. En el primero, encontramos que los estudiantes aplican la propiedad distributiva, obtienen una ecuación de segundo grado y a partir de ésta utilizan la fórmula resolvente para determinar los valores de $x$ que la anulan. Este procedimiento desemboca a la vez en otros dos posibles. Uno de ellos es utilizar los resultados obtenidos de aplicar la fórmula resolvente para luego abordar el estudio de la desigualdad a partir de analizar posibilidades según la variable tome valores menores, mayores o esté entre los dos ceros hallados. En este caso, consideramos que se interpreta la idea de inecuación. El otro procedimiento encontrado se reduce a resolver la ecuación asociada, obtener los dos valores que anulan la expresión y terminar la resolución. Pareciera que la solución de la ecuación se asocia con la de la inecuación o, en caso de que no ocurra esto, no queda expresado. Esto último sucede aún en los casos en los que al comienzo tienen en cuenta la desigualdad, como se encontró en cuatro de los estudiantes.

El segundo tratamiento general, no usa la ecuación de segundo grado. En este caso, encontramos el que toma en cuenta los factores involucrados en la inecuación y estudia las posibilidades de sus signos para que se cumpla la desigualdad. Ocho estudiantes plantean las distintas posibilidades de los signos de los factores del producto para que la desigualdad sea menor a cero. En este caso, obtienen -con o sin errores en las cuentas- el conjunto solución y lo expresan en términos de intervalos. En estos casos este tipo de tratamiento nos da la idea de que los estudiantes efectivamente logran alguna interpretación de la inecuación. Entendemos que aquí subyace un razonamiento de tipo aritmético, de análisis de signos. 
En el caso del ítem 1) b), resolver $\left|\frac{x}{4}-2\right| \geq 1$, pudimos determinar dos tipos de procedimientos entre quienes lo encararon. En uno, plantean usar la definición de valor absoluto considerando los casos en los que $\frac{x}{4}-2$ es mayor o igual que 0 , o menor; y el otro considera utilizar la propiedad (para $a>0$ ) $|x|>a \Leftrightarrow x>a \vee x<-a$. Independientemente de esta elección, se encuentran resoluciones correctas e incorrectas. Cabe señalar que once estudiantes cometen errores algebraicos al resolver el ejercicio y no llegan a la solución correcta. Sin embargo, interpretan el resultado que obtienen y presentan el conjunto solución de manera coherente con lo resuelto.

El ítem 1) c) es el que presentamos desarrollado en la fundamentación: resolver $7+$ $x^{2} \leq 7$. En este ejercicio distinguimos estudiantes que identifican que el único número que puede satisfacer la desigualdad es 0 y los que no. Dentro de los primeros, encontramos por ejemplo que un estudiante escribe el enunciado e inmediatamente agrega "se cumple para $x=$ 0". También podemos ver que, aun identificando la solución, realizan algún tratamiento algebraico. Se observa también la manipulación algebraica en primera instancia y luego la conversión al registro verbal para explicar lo hallado. Esto puede verse por ejemplo cuando luego de la resolución analítica, queda expresado "Solo en los casos que $x$ sea igual a cero, ya que analizando el cuadrado de un número nunca será negativo para que se cumpla la desigualdad pero si cero para que se cumpla la igualdad".

Se privilegia notablemente el tratamiento algebraico por más que no sea necesario, encontrando solo seis estudiantes que expresan verbalmente que solo 0 puede cumplir esa condición.

Finalmente, el ítem 1 d) plantea resolver $5-x<x-1$. Este ítem presenta una inecuación similar a las que tradicionalmente se proponen como los primeros ejemplos en clases. $\mathrm{Al}$ analizar las resoluciones de los estudiantes, encontramos que se repite en todos los casos el tratamiento dentro de ese registro para obtener el conjunto solución.

En el ejercicio 2 (resolver $\operatorname{sen} x \leq 1$ y $\operatorname{sen} x \geq 1$ ) fueron 18 los estudiantes que plantean posibles conjuntos solución mostrando que interpretaron lo que se les pidió, independientemente que resultara o no correcta su respuesta. El resto no resuelve. Sin embargo, las argumentaciones que presentan son dispares. Dejamos a continuación algunas respuestas encontradas. Un estudiante dibuja una circunferencia trigonométrica y coloca en el eje de 
abscisas valores del dominio como $0, \frac{\pi}{2} \cdot \frac{3 \pi}{2}, \pi$ y presenta su conjunto solución. Otro alumno encara la resolución de sen $x \leq 1$ como si estuviera resolviendo una ecuación y escribe: $x \leq$ $\operatorname{sex}^{-1}(1), x \leq 90,([-\infty] ; 90)=\left(-\infty ; \frac{1}{2} \pi\right)$ mientras que para $\operatorname{sen} x \geq 1$ deja escrito $x \geq$ $90^{\circ}, x \geq \frac{1}{2} \pi,\left[\frac{1}{2} \pi ;+\infty\right]$. Encontramos respuestas como: “con calculadora probé porque no sé cómo debería calcularlo", dejando en evidencia el uso del registro numérico.

El ejercicio 3 pide resolver dos inecuaciones. Ambas fueron planteadas a partir de dos gráficos cartesianos. Cada uno de ellos presenta el gráfico de dos funciones de valores reales que se intersecan. Uno de los casos fue diseñado de modo que los estudiantes pudieran reconstruir las expresiones algebraicas de las funciones cuyos gráficos presentamos. La otra inecuación, sin embargo, no admitía esta posibilidad. Este es el ejercicio con menor cantidad de respuestas. Solo 15 estudiantes lo realizan. El resto no escribe nada. Son dos los estudiantes que intentan reconstruir las expresiones algebraicas de las funciones, no logran destrabar el manejo algebraico y la resolución queda trunca. Un único estudiante deja de manifiesto que interpreta la condición de desigualdad y cuál es el conjunto solución, aunque de manera incorrecta y sin justificación. Hubo casos que resuelven o justifican en el plano dejando sombreado el subconjunto sobre el eje de las $x$. Finalmente señalamos un estudiante que deja expresado: "no puedo hacerlo, no se resolver inecuaciones de funciones". Suponemos que la representación de las inecuaciones en el registro gráfico por un lado podría haber obstaculizado su reconocimiento como objeto conocido, al no encontrarlas en el registro predominante de trabajo: el algebraico. Por otro lado, le hizo considerar que son un nuevo objeto matemático sobre el que no trabajó y por eso no puede resolver.

Los ejercicios 4 y 5 plantean dos situaciones en contexto extramatemático, una referida a ganancias de dos fábricas en función de ventas y la otra, a edades de padre e hijo. La primera de ellas presentó la mayor cantidad de respuestas, solo dos de los estudiantes no resolvieron. El procedimiento predominante fue realizar una conversión del registro verbal al numérico y realizar tratamientos obteniendo valores para cada una de las fábricas y luego compararlos. Otra resolución realizada fue mediante una conversión al registro algebraico, intentando plantear un sistema de ecuaciones lineales que les permitiera obtener el valor que verifica las dos igualdades asociadas. Dos estudiantes realizan esto correctamente y la mayoría responde adecuadamente al planteo de la inecuación a partir del valor hallado. 
El ejercicio de las edades es encarado, mayoritariamente a través de una conversión del registro verbal en el que fue planteado, al algebraico y solo un estudiante trabaja directamente en el registro numérico. El ejercicio propone encontrar un período en el que la edad del padre cumple una cierta condición, lo que implica que el conjunto solución debería ser un intervalo de tiempo. Los estudiantes plantean un sistema de ecuaciones que les permite hallar edades a partir de las cuales construir la respuesta a la inecuación. Cometen errores algebraicos o de planteo y es luego de esa primera conversión que apelan al registro numérico. Los errores les impiden avanzar en la resolución. Cabe resaltar que los valores dados en el enunciado posibilitan, por medio de prueba y error, hallar numéricamente datos que les permitieran responder sin necesidad de apelar a otro registro que no fuera el numérico.

El ejercicio 6 plantea: ¿Es posible que el cuadrado de un número real sea menor que el opuesto de dicho cuadrado? Explicar, y en caso de que sea posible, indicar todos los números que cumplen tal condición.

Siete estudiantes no resuelven y once usan el registro verbal para dar en conjunto solución y, en alguno de los casos, este último fue usado para justificar lo que escriben en el registro algebraico. Encontramos respuestas como "el cuadrado de todo número real es siempre positivo" o "no es posible ya que el cuadrado de un número siempre será positivo sin importar la base". También se presentan planteos algebraicos, correctos o no y la conversión del registro algebraico al numérico para intentar una forma de validación. Esto se dio en dieciocho casos. A modo de ejemplo: un estudiante escribe $x^{2}<(-x)^{2}$ y luego explica que "no es posible porque $(-2)^{2}$ sea menor que $2^{2}$, ya que $(-2)^{2}=4$ y $2^{2}=4$ y se obtiene el mismo número entero o el mismo resultado".

Finalmente, el ejercicio 7 plantea resolver la inecuación que corresponde a la positividad de una expresión homográfica. Doce estudiantes ni siquiera lo encaran y quienes sí lo hacen utilizan el registro algebraico en el que fue planteado. Quienes intentaron la resolución proponiendo "transposición de términos" (multiplicar miembro a miembro por el denominador), cometieron el error usual de no considerar los casos en que el signo del denominador es mayor o menor que cero. Sin embargo veinte estudiantes proponen el análisis de los signos de numerador y denominador por separado con desarrollos correctos o no. 
Aunque hemos analizado por separado los registros en los que los estudiantes presentaron los conjuntos solución de los siete ejercicios (como puede verse en Campos, 2020), presentamos aquí una síntesis global de los mismos.

En muchos casos encontramos que no presentan explícitamente el conjunto solución, simplemente "recuadran" una parte de su desarrollo. A veces ese recuadro expresa la resolución de la ecuación asociada a la inecuación. Otras, en cambio señala una desigualdad a partir de la cual ya no se puede operar simbólicamente $(x>3$, por ejemplo) pero no resulta claro que el estudiante comprenda que esa representación indica la totalidad de valores que satisfacen la inecuación, que conforman un intervalo de la recta real y que son todos los posibles. Encontramos, asimismo, representaciones en distintos registros. Muchos utilizan la notación de intervalo, o el sombreado de la región en la recta real. Los registros menos utilizados son el verbal y el numérico, alineado con los ejemplos prototípicos de la enseñanza.

\section{Respecto de la aprehensión conceptual}

En esta sección presentamos resultados del análisis por estudiante. El mismo nos permite expresar una valoración respecto de la aprehensión conceptual individual alcanzada. Primeramente mencionamos características comunes que hemos advertido en el desempeño de los estudiantes y que proponemos tras un proceso de síntesis que siguió a uno de tipo analítico. Partimos de resoluciones de distintos estudiantes y logramos establecer rasgos generales referidos a la aprehensión conceptual respecto de las inecuaciones que nos permitió agruparlos. Proponemos un nombre identificatorio para cada grupo de estudiantes, según nuestra apreciación, de la característica que consideramos más relevante que ponen de manifiesto: manipuladores simbólicos, manipuladores algebraicos y resolutores de inecuaciones.

Para cada uno de los grupos presentamos primero los rasgos que le son propios y luego los vinculamos con la aprehensión conceptual alcanzada de los sujetos que los conforman. Finalmente mostramos muy brevemente ejemplos de cada uno de ellos.

\section{Rasgos comunes a cada grupo}

Presentamos los rasgos organizados en tres grupos, y lo hacemos con dos salvedades. Primeramente, cabe aclarar que no hay pretensión de exhaustividad y extender o ajustar el listado de rasgos queda como asunto abierto. En segundo lugar, no todos los rasgos los hemos advertido en un mismo sujeto. 


\section{Manipulador simbólico}

Algunas características comunes son las siguientes:

- Se advierte manipulación algebraica pero no se percibe su finalidad.

- Su manipulación algebraica manifiesta contradicciones que no son advertidas por el sujeto.

- Se encuentra manipulación algebraica innecesaria, denotando falta de claridad en lo que se resuelve.

- Se encara la resolución de cualquier inecuación en el plano algebraico, sea productivo, o no. No se manifiesta abandonar los símbolos si no son efectivos.

- Ante consignas que se presentan similares a otras "prototípicas", entendidas estas últimas como aquellas que, escolarmente, suelen asociarse con un tipo de resolución o que conllevan un contenido matemático específico que el estudiante reconoce al verlas, se elige ese tipo de resolución o contenido, aunque no sea lo pedido.

- Se realiza algebraicamente algún procedimiento y, al terminarlo, no se presenta respuesta a la consigna.

Vínculo entre estudiantes de este grupo y su aprehensión conceptual del objeto inecuaciones: el hecho de manipular símbolos sea o no productivo, no advertir las contradicciones que el mismo sujeto provoca, hace que quede de manifiesto que su manejo algebraico no está suficientemente desarrollado. Si, además, agregamos que no apela a otras representaciones pues ante cualquier inecuación la aborda mediante símbolos, no deja de manifiesto la utilización adecuada de al menos dos registros de representación semiótica, lo que nos permite concluir que los estudiantes de este grupo no alcanzan una aprehensión conceptual del objeto inecuaciones.

\section{Manipulador algebraico}

En estos estudiantes encontramos rasgos que son comunes al grupo 1 y otros particulares que los diferencian, fundamentalmente por el avance en la manipulación algebraica, ya con un objetivo definido.

Algunas características comunes son los siguientes:

- Se advierte manipulación algebraica con la finalidad de obtener una solución a la inecuación. 
- Se encuentran resoluciones en registros diferentes al algebraico, como el gráfico en el plano y verbal.

- Los conjuntos solución son expresados en los registros algebraicos con notación de conjuntos, gráficos en la recta real, notación de intervalos en casi todos los casos sintácticamente incorrectos.

- Su manipulación algebraica manifiesta contradicciones que no son advertidas por el sujeto.

- Ante consignas que se presentan similares a otras "prototípicas", actúa de igual modo que lo mencionado en el caso del manipulador simbólico.

- Se encuentra manipulación algebraica innecesaria, denotando falta de claridad en lo que se resuelve.

Vínculo entre estudiantes de este grupo y su aprehensión conceptual del objeto inecuaciones: el hecho de manipular símbolos con el objetivo de obtener una posible solución y la utilización de al menos dos tipos de registro nos muestra un grado mayor, respecto de los estudiantes del primer grupo, de aprehensión conceptual del objeto inecuaciones.

\section{$\underline{\text { Resolutor de inecuaciones }}$}

Aunque se presentan errores en los tratamientos y en algunos casos no pueden advertir las contradicciones que ellos mismos provocan, queda de manifiesto un manejo algebraico mejor desarrollado que el de los estudiantes del grupo 2.

Tomamos como indicador para visualizar un claro avance hacia la resolución de inecuaciones las decisiones sobre los tratamientos algebraicos relacionados con la construcción y comunicación en alguno de los registros posibles un conjunto solución coherente con lo solicitado en el ejercicio.

Algunas características son los siguientes:

- Se advierte manipulación algebraica con la finalidad de obtener una solución a la inecuación.

- Realizan una conversión al registro numérico y determinar los que validan la desigualdad. Podemos decir que exploran numéricamente la inecuación.

- Los conjuntos solución son expresados en los registros algebraicos con notación de conjuntos, gráficos en la recta real, notación de intervalos en casi todos los 
casos sintácticamente incorrectos pero muestran que identifican valores del dominio que cumplen con la condición de la desigualdad.

- Realizan la conversión del registro gráfico al algebraico para poder realizar tratamientos en ese registro.

- Consideran el conjunto al que pertenecen los valores de la variable que permiten que se cumpla la desigualdad.

- Son los que escriben el conjunto solución sintácticamente más aproximado a lo correcto.

Vínculo entre estudiantes de este grupo y su aprehensión conceptual del objeto inecuaciones: en los estudiantes de este grupo encontramos tratamientos en el registro algebraico que superan lo que llamamos "manipulación algebraica", cuestión que se manifiesta en la escritura coherente de los posibles conjuntos solución, aunque no siempre resulten sintácticamente correctos.

Aquí también el hecho de manipular símbolos con el objetivo de obtener una posible solución y la utilización de al menos dos tipos de registros nos muestra un grado mayor, respecto de los estudiantes del grupo anterior, de aprehensión conceptual del objeto inecuaciones.

Aunque se presentan errores en los tratamientos y en algunos casos no pueden advertir las contradicciones que ellos mismos provocan, queda de manifiesto un manejo algebraico mejor desarrollado que el de los estudiantes del grupo 2.

\section{Ejemplos de resoluciones correspondientes a estudiantes de cada grupo}

Algunos desempeños de estudiantes que consideramos dentro del grupo manipulador simbólico son las siguientes.

Al resolver $2(x-1)(x+2)<0$ encontramos el desarrollo de la multiplicación indicada, la construcción de la ecuación de segundo grado asociada y aplicación de la fórmula resolvente. En muchos casos ahí termina su resolución. Otros, obtienen valores erróneos de la ecuación sin asociarlos con que el planteo los deja a la vista por tener que anular alguno de los

factores. Ante la inecuación $\left|\frac{x}{4}-2\right| \geq 1$ estudiantes de este grupo solo toman una de las posibilidades $\left(\frac{x}{4}-2 \geq 1\right)$, sin considerar la restricción que se debe imponer para llegar a ella 
$\left(\frac{x}{4}-2 \geq 0\right)$, obtienen $x \geq 12$ y recuadran ese resultado. Para el ejercicio $7+x^{2} \leq 7$ se encuentran tratamientos algebraicos y respuestas como: $x \leq 0$ o " $x \leq \sqrt{0}$ no tiene solución".

Para la inecuación $\frac{3 x-9}{2 x+8}>0$ encontramos resoluciones como:

$3 x-9>(2 x+8) .0,3 x-9>0$. Vemos que no considera que no es posible multiplicar miembro a miembro una desigualdad por un factor si se desconoce su signo y que este hecho le obligaría a imponer condiciones, en este caso sobre el denominador. Obtiene, luego de realizar tratamiento algebraico, $x>3$. Opera de igual modo que si tuviera una ecuación.

Algunos desempeños de estudiantes que consideramos dentro del grupo manipulador algebraico son las siguientes.

Encontramos resoluciones en el registro algebraico y un intento de expresar el conjunto solución.

Ante la inecuación $2(x-1)(x+2)<0$, al igual que en el ejemplo anterior, construyen una ecuación de segundo grado y aplican la fórmula resolvente obteniendo dos valores para $x$, $x=-2$ y $x=1$. Sin embargo se ven respuestas como $S=\{x \in \mathbb{R} /-2<x<1\}$, o la representación del intervalo en la recta real.

Para el ejercicio $7+x^{2} \leq 7$ los estudiantes de este grupo realizan tratamientos algebraicos y obtienen $x \leq 0$, a diferencia del grupo anterior expresan el conjunto solución de alguna manera, por ejemplo un estudiante escribe $S=\{x \leq 0\}$ y representa en el gráfico en la recta real destacando la semirrecta que corresponde a valores de $x \leq 0$.

Algunos desempeños de estudiantes que consideramos dentro del grupo resolutores de inecuaciones son las siguientes.

Casi todos los estudiantes que ubicamos dentro de este grupo resuelven el ejercicio 1) completo.

Ante el ejercicio que plantea resolver $\operatorname{sen} x \leq 1$ y sen $x \geq 1$ responden proponiendo conjuntos solución en el registro verbal y en el algebraico. Algunos fundamentan sus respuestas explicando los valores posibles de la variable $x$ para que se cumplan las desigualdades. Por ejemplo, encontramos respuestas como "el sen $x$ siempre te va dar entre $1 \mathrm{y}-1$ ", para el ejercicio b) luego de dar dos ejemplos $x=90^{\circ} \rightarrow \operatorname{sen} x=1$ y $x=450^{\circ} \rightarrow \operatorname{sen} x=1$, escribe para generalizar "mientras caiga en el límite del primer cuadrante siempre te dará igual a 1". Otros diez estudiantes proponen soluciones más completas y muy aproximadas a las correctas. 
Otro estudiante escribe para a) "conjunto solución para las inecuaciones $\operatorname{sen} x \leq 1$ solución $=$ $\mathbb{R}$ ” y "sen $x \geq 1$ solución $=\frac{\pi}{2}+2 k \pi$ con $k \in \mathbb{Z}$ ". También encontramos "la función sen oscila entre $-1 y 1$, por lo tanto para cualquier $x \in \mathbb{R}$ cumple con la inecuación" y para b) "la

función seno es igual a 1 si y solo sí $x=\frac{\pi}{2}$ y se repite cada $2 \pi, S=x \in \mathbb{R} \wedge k \in \mathbb{N}_{0} / x=$ $2 k \frac{\pi}{2}$

En el planteo de las inecuaciones por medio de gráficas de funciones en el plano cartesiano, ocho estudiantes lograron, de la lectura del gráfico, identificar los puntos de intersección de las funciones propuestas y determinan el conjunto solución. Para el ejercicio 5) sobre edades de personas, los estudiantes de este grupo reconocen que deben encontrar un período de tiempo y que sus respuestas deben mostrar de alguna manera un intervalo en alguno de los registros posibles.

\section{Consecuencias del análisis}

Luego del estudio que nos permitió realizar un análisis por estudiante y tras la propuesta de agrupamiento, construimos una rúbrica que consta de cuatro dimensiones, vinculadas a la resolución de inecuaciones, con una identificación en tres etapas: inicial, intermedia y avanzada que plasman distintos logros de aprehensión conceptual.

Aunque concebimos las etapas como parte de un proceso continuo, para que resulten operativas las presentamos de manera discreta. Aquí quedan expresadas en las tres columnas de la tabla.

Las cuatro dimensiones mencionadas pueden ser consideradas en la aprehensión conceptual de otras temáticas del álgebra, sin embargo las proponemos aquí dado que son cuestiones clave al trabajar con inecuaciones.

En cada celda de la primera y de la tercera columna pueden verse indicadores que se corresponden con la clasificación de los grupos del apartado anterior. Para establecer en qué etapa de aprehensión conceptual sobre inecuaciones se encuentra un sujeto, tal como hemos mencionado, no es necesario que presente la totalidad de las características que allí se expresan.

De este modo, la rúbrica que expresa distintos alcances en la aprehensión conceptual se muestra en el Cuadro 1. 
Cuadro 1.Rúbrica para valorar la aprehensión conceptual de inecuaciones

\begin{tabular}{|c|c|c|}
\hline $\begin{array}{c}\text { Etapa inicial } \\
\text { Grupo } 1 \\
\end{array}$ & $\begin{array}{c}\text { Etapa intermedia } \\
\text { Grupo 2 } \\
\end{array}$ & $\begin{array}{c}\text { Etapa avanzada } \\
\text { Grupo } 3 \\
\end{array}$ \\
\hline \multicolumn{3}{|c|}{ SOBRE LA MANIPULACIÓN SIMBÓLICA } \\
\hline $\begin{array}{l}\text { Manipula innecesariamente } \\
\text { / No se advierte la finalidad } \\
\text { / Presenta contradicciones / } \\
\text { No advierte sus } \\
\text { contradicciones / opera } \\
\text { correctamente o no }\end{array}$ & $\begin{array}{l}\text { Se encuentra un avance } \\
\text { de lo descripto a la } \\
\text { izquierda, en dirección } \\
\text { a los rasgos que indican } \\
\text { un mayor desarrollo }\end{array}$ & $\begin{array}{l}\text { Manipula simbólicamente } \\
\text { cuando es necesario / } \\
\text { Advierte la finalidad / No } \\
\text { presenta contradicciones / } \\
\text { Opera correctamente } \\
\text { (desde lo matemático) }\end{array}$ \\
\hline \multicolumn{3}{|c|}{ SOBRE EL USO DE DISTINTOS REGISTROS } \\
\hline $\begin{array}{l}\text { Solo apela al álgebra / No } \\
\text { abandona el álgebra / } \\
\text { considera que las } \\
\text { inecuaciones deben } \\
\text { resolverse algebraicamente } \\
\text { y fuerza la aparición del } \\
\text { álgebra }\end{array}$ & $\begin{array}{l}\text { Se encuentra un avance } \\
\text { de lo descripto a la } \\
\text { izquierda, en dirección } \\
\text { a los rasgos que indican } \\
\text { un mayor desarrollo }\end{array}$ & $\begin{array}{l}\text { Selecciona el registro que } \\
\text { considera adecuado para } \\
\text { resolver / Resuelve } \\
\text { inecuaciones en distintos } \\
\text { registros / Utiliza distintos } \\
\text { registros para verificar }\end{array}$ \\
\hline \multicolumn{3}{|c|}{ SOBRE EL CONJUNTO SOLUCIÓN Y LA RESPUESTA A LA CONSIGNA } \\
\hline $\begin{array}{l}\text { No es claro cuál es / se } \\
\text { encuentra tratamiento } \\
\text { algebraico sin } \\
\text { identificación de respuesta } \\
\text { o se advierte tratamiento } \\
\text { algebraico y al finalizar una } \\
\text { identificación -no explícita- } \\
\text { que pareciera una respuesta } \\
\text { (en general se ve alguna } \\
\text { parte "recuadrada") }\end{array}$ & $\begin{array}{l}\text { Se encuentra un avance } \\
\text { de lo descripto a la } \\
\text { izquierda, en dirección } \\
\text { a los rasgos que indican } \\
\text { un mayor desarrollo }\end{array}$ & $\begin{array}{l}\text { Está identificado el } \\
\text { conjunto solución } \\
\text { claramente / es coherente } \\
\text { con lo hallado (sea o no } \\
\text { matemáticamente correcto) } \\
\text { / Puede encontrarse el } \\
\text { conjunto solución } \\
\text { expresado en distintos } \\
\text { registros, según la } \\
\text { resolución }\end{array}$ \\
\hline \multicolumn{3}{|c|}{ SOBRE LOS ENUNCIADOS } \\
\hline $\begin{array}{l}\text { Trata inecuaciones como } \\
\text { ecuaciones / Hace analogías } \\
\text { con ejercicios prototípicos } \\
\text { de otros temas / Prueba } \\
\text { numéricamente en } \\
\text { enunciados dados en } \\
\text { registro verbal }\end{array}$ & $\begin{array}{l}\text { Se encuentra un avance } \\
\text { de lo descripto a la } \\
\text { izquierda, en dirección } \\
\text { a los rasgos que indican } \\
\text { un mayor desarrollo }\end{array}$ & $\begin{array}{l}\text { Reconoce, a partir de } \\
\text { enunciados en distintos } \\
\text { registros, que está ante una } \\
\text { inecuación }\end{array}$ \\
\hline
\end{tabular}

Fuente: elaborado por las autoras

\section{Discusión}

El análisis que realizamos nos permitió rastrillar los datos recabados en el siguiente sentido. Por un lado, logramos entender los registros que los estudiantes ponen en juego ante la resolución de cada inecuación planteada, con sus particularidades en función del registro en el 
que se les presentó. Pero, complementariamente, hemos podido analizar el desempeño de cada estudiante, lo que nos llevó a proponer agrupamientos de ellos en función del modo en el que se desenvuelven ante la resolución de inecuaciones.

Para ampliar lo que cada actividad, individualmente, nos ha permitido entender mencionamos las siguientes cuestiones. Hemos presentado inecuaciones que no requieren de ningún tipo de tratamiento ni conversión para su resolución. Para resolverlas, solo es necesario comprender lo que ese planteo simbólico representa. Este hecho no fue advertido, en general, por los estudiantes quienes se embarcaron en manipulaciones simbólicas, correctas o no, que los llevaron a responder o ni siquiera intentarlo. También presentamos enunciados similares a otros que los estudiantes han trabajado a propósito de otros contenidos matemáticos. En este caso, hemos visto que esa similitud en apariencia los llevó a no reconocer la inecuación subyacente $\mathrm{y}$, en cambio, retomar aquellos otros contenidos y resoluciones que le fueron eficaces anteriormente. Reconocemos, a partir de este tipo de desempeño, que aún falta que los estudiantes analicen qué es lo que se les solicita así como lo que responden, tengan en claro cuál es el alcance de los procedimientos que utilizan en relación a tal o cual objeto matemático, como así también la validez de su aplicación, o qué objetos matemáticos son útiles o no para responder a una situación específica.

En la mirada complementaria a la recién mencionada, respecto de la aprehensión conceptual alcanzada por cada estudiante, pudimos reconocer tipos de respuestas matemáticas similares que, de alguna manera, nos permiten advertir rasgos comunes a grupos de estudiantes que hemos propuesto asociar con niveles en el aprendizaje de las inecuaciones. Como señalamos en breve, entendemos que esto nos habilita a delinear un trabajo en el plano docente que nos permitirá tener herramientas para mejorar las propuestas de enseñanza. Pero, antes de ir en esa dirección, queremos mencionar algunas reflexiones de lo que hemos estudiado.

Nos encontramos con un grupo de estudiantes que manipula símbolos -correctamente o no- sin comprender la finalidad, ni entender qué significa aquello que logra tras la manipulación. Estos estudiantes pareciera que operasen por un tipo de inercia que, al ver un planteo simbólico -más allá de cuál fuera- los lleva a manipular, operar. Asociado a esto, como en la escolaridad previa las ecuaciones tuvieron su presencia primeramente, muchos de estos estudiantes toman las inecuaciones como si fueran ecuaciones. No es claro si la familiaridad del planteo (se "ve casi igual", excepto porque el símbolo = no está) los lleva a las ecuaciones o si "ver símbolos" 
los lleva a manipularlos y realizar tratamientos similares en el registro en que fue planteado. Esto queda en consonancia con lo que Garrote, Hidalgo y Blanco (2004) mencionan sobre los estudiantes, señalando que no establecen diferencias significativas entre este concepto y el de ecuación y que, por lo tanto, no logran comprenderlo.

También encontramos variedad de matices entre quienes están en el proceso de aprendizaje de las inecuaciones y han logrado trascender esta "manipulación simbólica" como un fin en sí mismo, pero que aún no alcanzan la aprehensión conceptual esperada.

Un punto clave que la teoría señala y que hemos encontrado con total claridad es la presencia, o falta, de la actividad cognitiva de conversión, clave para lograr la aprehensión conceptual. Encontramos muchas dificultades en que los estudiantes acudan a representaciones en otro registro, que no sea el simbólico. Esto obturó, además de la posibilidad de conversión, la resolución de inecuaciones cuyo planteo no estuvo dado en registro algebraico. Es notable esta situación dado que estos estudiantes estarían en lo que Guzmán (1988) llama monoregistro: manipulan una sola forma de representación y no pueden coordinar diferentes tipos de registros para un mismo objeto matemático. Esto nos permite concluir la no aprehensión conceptual.

También resaltamos como importante la falta de reconocimiento del conjunto solución de las inecuaciones. El hecho de operar simbólicamente y no responder, no advertir contradicciones o recuadrar un resultado parcial son muestras de que los estudiantes no responden en relación con la pregunta que la actividad plantea, dejando de manifiesto que no se ha alcanzado la comprensión pretendida. Esta ausencia de coordinación entre los diferentes registros genera un obstáculo para el aprendizaje, en este caso particular, de las inecuaciones.

Queremos retomar aquí el problema docente de un modo reflexivo. Podríamos explicar “desde las tradiciones de la enseñanza" que las inecuaciones suelen trabajarse casi exclusivamente desde planteos algebraicos. Asimismo, hemos advertido en la bibliografía consultada, en libros de texto y en materiales didácticos que no es usual el trabajo con inecuaciones que se presenten en registro gráfico, numérico o verbal. Queremos, a continuación, proponer posibles explicaciones a estos dos últimos casos.

Respecto del registro numérico, cabe señalar que cuando el conjunto solución admite infinitos valores que forman un conjunto no numerable, no resulta posible manejarse exclusivamente en ese registro para expresar esa totalidad. Cuando el conjunto solución es infinito numerable o finito, sería factible dejar indicado, con una tendencia, cuáles serían esos 
valores o exhibirlos exhaustivamente (en el caso finito, si la cantidad fuera razonable). Esto marca una dificultad en el uso de este registro y tal vez sea, en alguna media, una causa de que no se use para resolver, ni para plantear inecuaciones, pero sí en cambio sea utilizado para explorar y/o verificar. Señalamos también, que casi nunca es posible verificar que el conjunto solución hallado sea tal. Un error muy común aquí es considerar que hemos verificado la solución de una inecuación pues hemos chequeado que un par de valores hallados, la satisfacen. Esto no es suficiente pues queda sin considerarse la posibilidad de que haya otros valores que la cumplen $\mathrm{u}$ otras supuestas soluciones que no lo sean. Lo mismo suele ocurrir con las ecuaciones. Esto suele ser un planteo equivocado que está presente en la escolaridad y que se traslada a este nuevo contenido. Es decir, no es correcto el procedimiento escolarizado de esperar que un alumno resuelva una ecuación, halle una cierta cantidad de valores, los pruebe evaluando, o pruebe "alguno" de ellos, obtenga un resultado verdadero y considere que seguro ha resuelto correctamente la ecuación.

Respecto de la ausencia del registro verbal al trabajar con inecuaciones, conjeturamos que podría deberse a que, en general, cuando éste se utiliza coloquialmente, las inecuaciones expresan casi inmediatamente condiciones triviales, como los siguientes ejemplos señalan. Cuando "seas mayor de 18 años, podrás manejar", "ganarás entre \$100 y \$300 por cada venta", la velocidad mínima es de $50 \mathrm{~km} / \mathrm{h}$ en autopista y la máxima de $110 \mathrm{~km} / \mathrm{h}$ ). Estos ejemplos permiten ver que no hay necesidad aquí de resolver una inecuación. Entendemos que estos planteos se vinculan más con el objeto subconjuntos numéricos o intervalos reales. Es decir, una representación $100<x<300$ por un lado ciertamente plantea una inecuación pero es más utilizada para representar un subconjunto numérico, un intervalo real. La escolaridad media genera la tradición que las ecuaciones, y también las inecuaciones, necesitan trabajarse, manipularse.

Ahora bien, quedarse con un solo registro, el algebraico, por más que sea dúctil y el estudiante se manejara cómodamente en él, sabemos que no basta para logar la aprehensión conceptual del objeto en cuestión pues deberían articularse al menos dos registros. Entonces, dado que los registros de representación semiótica son el verbal, numérico, algebraico y gráfico; los dos primeros son débiles para este objeto y el algebraico es el predominante, el único camino que quedaría habilitado para que desde la enseñanza se pudiera promover la aprehensión conceptual, es forzar el trabajo con las inecuaciones en el registro gráfico y promover la toma 
de decisiones de en cuál registro trabajar, según el planteo dado. Consideramos que esta conclusión brinda herramientas potentes para el trabajo docente pues advierte el tipo de planteo y discusión que sería imprescindible incluir en las clases. De este modo, consideramos que podría transferirse, a la enseñanza, alguno de los resultados y conclusiones que se alcanzaron tras llevar adelante la investigación aquí presentada.

Señalamos también una cuestión importante a tener en cuenta desde la perspectiva de la enseñanza. Hemos visto que un estudiante podría resolver inecuaciones con solvencia en registro algebraico únicamente y no haber alcanzado la aprehensión conceptual. Esto ocurriría cuando el estudiante no maneja ninguna representación en otro registro y podría deberse a que la enseñanza le plantea, con casi exclusiva predominancia, resolver inecuaciones presentadas en registro algebraico. Ahora bien, si este es el caso, y si el docente plantea únicamente inecuaciones para ser resueltas algebraicamente, y se encuentra con un buen desempeño del estudiante ambos -docente y estudiante- podrían creer que ha comprendido el tema. En este caso, quedaría oculto para ambos que esa ductilidad algebraica podría no ser suficiente indicador de comprensión pues podría solo manejarse en ese registro. Si el docente le preguntara al estudiante de otro modo, o le presentara inecuaciones en registro gráfico se vería si es capaz de articular otro registro o no. Ahora bien, el caso es distinto si el estudiante resuelve mal, porque entonces queda en evidencia que no ha comprendido. Por otra parte, un estudiante podría tener un acercamiento a la aprehensión conceptual y pasar desapercibida por ambos, a saber. Podría no resolver correctamente, en registro algebraico pero entender la idea, entender qué es lo que debe resolver, cómo debe ser el tipo de respuesta que debe dar, etc. Este es un caso en el que usualmente su profesor podría no darse cuenta de que el estudiante sí tiene una aproximación al concepto y calificaría como incorrecta su resolución, quedando como síntesis que no sabe inecuaciones.

\section{Referencias}

Alvarenga, K. (2005). Un análisis de las construcciones mentales de estudiantes universitarios, México DF, México, Centro de Investigación en Ciencia Aplicada y Tecnología Avanzada, Instituto Politécnico Nacional.

Campos, M. (2020). Sobre la aprehensión conceptual de las inecuaciones. Un estudio en primer año del nivel superior. [Tesis de maestría], Universidad Nacional de La Rioja, Argentina. 
Aznar, M., Distéfano, M., Figueroa, A. \& Moler, E. (2010). Análisis de conversiones entre representaciones semióticas de números complejos [Archivo PDF], Recuperado de http://repem.exactas.unlpam.edu.ar/cdrepem10/memorias/comunicaciones/Trabajos\%20In ves/CB\%2021.pdf.

D’Amore, B., Fandiño, M., Iori, M. \& Matteuzzi, M. (2015). Análisis de los antecedente históricos-filosóficos de la "Paradoja cognitiva de Duval". Revista Latinoamericana de Investigación en Matemática Educativa, pp.177-212.

Duval, R. (1998). Registros de representación semiótica y funcionamiento cognitivo del pensamiento. Investigaciones en Matemática Educativa II (173-201). Ciudad de México. México: Grupo Editorial Iberoamericano.

Duval, R. (2004). Semiosis y pensamiento humano, Cali, Colombia, Instituto de Educación y Pedagogía de la Univesidad del Valle.

Duval, R. (2006). Un tema crucial en la educación matemática: La habilidad para cambiar de regsitro de representación. Gaceta de la Real Sociedad Matemática Española, 9 (1), pp.143168.

Duval, R. (2016). Comprensión y aprendizaje en matemáticas:perspectivas semióticas seleccionadas. Bogotá, Colombia, Universidad Distrital Francisco José Caldas.

Garrote, M. \& Hidalgo, M. (2004). Dificultades en el aprendizaje de las desigualdades e inecuaciones. Suma 46, pp.37-44.

Gatica, N. \& Maz Machado, A. (2012). Estudio de las inecuaciones de dos variables [Archivo PDF]. Recuperado de https://thales.cica.es/xivceam/actas/pdf/com05.pdf.

Guzmán, I. (1998). Registros de representación, el aprendizaje de las nociones relaticas a funciones:voces de los estudiantes. Relime, 1(1), pp.5-21.

Macías, S. (2016). Diseño y estudio de situaciones didácticas que favorecen el trabajo con registros semióticos [Tesis doctoral], Universidad Complutense de Madrid, España.

Moreno, J. \& Laborde, C. (2003). Articulation entre cadres et registres de représentation des équations différentielles dans un environnement de géométrie dynamique, Actes du Congrès Européen, ITEM, Reims, Francia.

Panizza, M. (2018). Las transformaciones semióticas en los procesos de definición de objetos matemáticos. [Tesis doctoral], Universidad Nacional de Córdoba, Argentina.

Vancken, S., Engler, A. \& Muller, D. (2010). Inecuaciones Algebraicas. Una experiencia didáctica articulando diversos sistemas de representación. Recuperado de https://www.researchgate.net/publication/269920192_Inecuaciones_Algebraicas_Una_Ex periencia_Didactica_Articulando_Diversos_Sistemas_de_Representacion. 